Pacific Journal of Mathematics

IMAGES OF MEASURABLE SETS

ilion Bressler and A. P. Morse 


\title{
IMAGES OF MEASURABLE SETS
}

\author{
D. W. Bressler and A. P. Morse
}

For a finitely additive and countably multiplicative family $H$, Measurable $H$ is the family of all sets which are measurable by every Carathéodory outer measure by which the members of $H$ are measurable and complements of members of $H$ are approximable from within. A relation contained in a topological product space is subvalent, if for some countable ordinal $\alpha$, each horizontal section of the relation has an empty derived set of order $\alpha$. A topological space is Borelcompact if it and the difference of any two of its closed compact subsets are countable unions of closed compact sets.

It is shown that if $X$ and $Y$ are Borelcompact, Hausdorff spaces with countable bases and $R$ is an analytic and subvalent subset of the cartesian product of $X$ with $Y$, then the direct $R$-image of $A$ is Measurable $\mathfrak{F}(Y)$ whenever $A$ is Measurable $\mathfrak{F}(X)$. ( $(\mathfrak{F}(X)$ is the family of closed subsets of $X$.) If $X$ and $Y$ are complete, separable, metric spaces and $R$ is an analytic and subvalent subset of $X \times Y$, the same conclusion can be drawn.

In a topological setting, the notion of measurability employed (Definitions 3.4.7 and 3.4.8) comprehends measurability by every Carathéodory outer measure by which closed sets are measurable and open sets can be approximated from within. More particularly if $\mathfrak{F}$ is the family of all real closed sets, then (3.4.8) Measurable $\mathfrak{F}$ is such a family that all real analytic sets belong to it and its members are Lebesgue measurable.

Because a topological setting sufficient for the current theory of analytic sets is required, the spaces concerned are either Borelcompact (Definition 4.13) Hausdorff spaces satisfying the second axiom of countability or complete, separable, metric spaces. Under these restrictions Souslin sets (Definition 3.2) and analytic sets (Definition 3.3) are the same, and a relation which is both Souslin and subvalent is the union of a countable family of relations which preserve measurability. This property of the component relations is obtained in Theorems 5.13 and 5.14. The decomposition of a subvalent relation (Theorem 7.6) is described by a transfinite operation of extraction (Definition 6.1) which is related to the familiar transfinite set derivation in topological spaces. The results announced in the introductory passage are contained in Theorems 8.3 and 8.4. 


\section{Notations.}

2.1. $\{A\}$ is the set whose sole member is $A$.

2.2. $\sigma A$ is the union of the members of $A$; i.e., $\sigma A=E t[t \in x$ for some $x \in A]$.

2.3. $A \sim B$ is the set of points in $A$ which are not in $B$.

2.4. $A \times B$ is the cartesian product of $A$ with $B$.

If $R$ is a relation (a set of ordered pairs) then

2.5. $\mathrm{dmn} R$ (domain $R$ ) is the set of first coordinates of pairs of $R$; i.e., $\mathrm{dmn} R=E x[(x, y) \in R$ for some $y]$,

2.6. $\operatorname{rng} R$ (range $R$ ) is the set of second coordinates of pairs of $R$; i.e., $\operatorname{rng} R=E y[(x, y) \in R$ for some $x]$.

If $R$ is a relation and $A$ is a set, then

2.7. ${ }_{*} R A$ is the direct $R$-image of $A$; i.e., ${ }_{*} R A=E y[(x, y) \in R$ for some $x \in A]$,

2.8. $* R A$ is the counter $R$-image of $A$; i.e., $* R A=E x[(x, y) \in R$ for some $y \in A]$,

2.9. $R \mid A$ is the restriction of $R$ to $A$; i.e., $R \mid A=E(x, y)[(x, y) \in R$ for some $x \in A]$.

2.10. $Q$ is the set of ordinals.

2.11. $\Omega$ is the set of countable ordinals; i.e., $\Omega$ is the least noncountable ordinal.

2.12. $\omega$ is the set of finite ordinals; i.e., $\omega$ is the least denumerable ordinal.

2.13. 0 is the least ordinal and the empty set.

2.14. $\bar{n}$ is the successor of $n$; i.e., $\bar{n}=n \cup\{n\}$.

Thus if $n \in \omega, \bar{n}=\operatorname{Em}[m \in \omega$ and $0 \leqq m \leqq n]$.

2.15. $S$ is the set of all sequences of natural numbers; i.e., $S$ is the set of all functions on $\omega$ to $\omega$.

2.16. $S_{n}^{\prime}$ is the set of all functions on $\bar{n}$ to $\omega$.

If $H$ is a family of sets, then 
2.17. $H_{\sigma}=E A\left[A=\bigcup_{n} \epsilon_{\omega} B_{n}\right.$ for some sequence $B$ of sets in $\left.H\right]$,

2.18. $H_{\delta}=E A\left[A=\bigcap_{n \in \omega} B_{n}\right.$ for some sequence $B$ of sets in $\left.H\right]$,

2.19. $H_{\sigma \delta}=\left(H_{\sigma}\right)_{\delta}$

2.20. $H_{\sim}=E A[A=\sigma H \sim B$ for some $B \in H]$.

3. Borel, Souslin, analytic and measurable sets.

3.1. Definitions. The Borel families.

3.1.1. Borelian $H$ is the smallest family which contains $H$ and is closed to countable, nonvacuous, union and intersection.

3.1.2. Borel ring $H$ is the smallest family which contains $H$ and is closed to countable union and set difference.

3.1.3. Borel field $H$ is the smallest family which contains $H$ and is closed to countable (including vacuous) union and complementation with respect to $\sigma H$.

3.2. Definition. Souslin sets. Souslin $H$ is the family of all sets $A$ such that

$$
A=\bigcup_{s \in S} \bigcap_{n \in \omega} h(s \mid \bar{n})
$$

for some function $h$ on $\bigcup_{n} \in_{\omega} S_{n}^{\prime}$ to $H$.

3.3. Definition. Analytic sets. Analytic in $X$ is the family of all sets $A$ such that $X$ is a topological space and

$$
A={ }_{*} f \alpha
$$

for some $\alpha \in K_{\sigma \delta}^{\prime}$ and some continuous function $f$ on $\alpha$ to $X$, where $K^{\prime}$ is the family of all closed, compact sets in a topological space $X^{\prime}$.

\subsection{Definitions. Measurable sets.}

3.4.1. $\varphi$ measures $X$ if and only if $\varphi$ is such a function on the subsets of $X$ that

$$
0 \leqq \varphi(A) \text { whenever } A \subset X
$$

and

$$
\varphi(A) \leqq \sum_{\alpha \in K} \varphi(\alpha)
$$


whenever $K$ is such a countable family that $A \subset \sigma K \subset X$. and

3.4.2. $\operatorname{mbl} \varphi($ measurable $\varphi)=E A[\varphi$ measures $\sigma \operatorname{dmn} \varphi, A \in \operatorname{dmn} \varphi$

$$
\varphi(T)=\varphi(T \cap A)+\varphi(T \sim A)
$$

whenever $T \in \mathrm{dmn} \varphi]$.

3.4.3. section $\varphi T$ is the function $\psi$ on $\operatorname{dmn} \varphi$ such that, for each $A \in \mathrm{dmn} \varphi$,

$$
\psi(A)=\varphi(T \cap A) .
$$

3.4.4. submeasures $\varphi=E \psi[\varphi$ measures $\sigma$ dmn $\varphi$ and $\psi=$ section $\varphi T$ for some $T \in \operatorname{dmn} \varphi$ such that $\varphi(T)<\infty]$.

3.4.5. $H$ is internal if and only if $H$ is closed to finite (including vacuous) union and nonvacuous countable intersection.

3.4.6. $\operatorname{prxn} \varphi H=E A\left[\varphi\right.$ measures $\sigma \operatorname{dmn} \varphi$ and $\left.\inf _{\sigma \in H} \varphi_{\partial \subset A}(A \sim C)=0\right]$.

Thus prxn $\varphi H$ consists of those sets which can be $\varphi$-approximated from the inside by members of $H$.

3.4.7. Mass $H=E \varphi[\varphi$ measures $\sigma H, H$ is internal, $H \subset \mathrm{mbl} \varphi$ and $H_{\sim} \subset \operatorname{prxn} \psi H$ whenever $\psi \in$ submeasures $\left.\varphi\right]$.

3.4.8. Measurable $H$ is the family of all sets $A$ contained in $\sigma H$ and such that $A \in \operatorname{mbl} \varphi$ whenever $\varphi \in$ Mass $H$.

4. Relations between the several families. In this section we assemble, in a convenient form, an account of some relations between Borel, Souslin and analytic sets in a topological setting.

Theorems 4.3, 4.4 and 4.10 are quite well known and are due to Lusin, Sierpiński and Souslin [4, 5, 9]. Theorems 4.5, 4.6, 4.7 and 4.8 are recent results of Choquet and Sion [3, 7]. For detailed references and some proofs the reader is referred to [2].

4.1. Notations. If $X$ is a topological space, then

4.1.1. $K(X)$ is the family of all closed, compact sets in $X$,

4.1.2. $\mathfrak{F}(X)$ is the family of all closed sets in $X$,

4.1.3. $\mathfrak{G}(X)$ is the family of all open sets in $X$,

4.1.4. $K_{\sigma}(X)=(K(X))_{\sigma}$. 
4.2. Theorem. $H \subset$ Borelian $H \subset$ Borel ring $H \subset$ Borel field $H \subset$ Measurable $H$.

4.3. THEOREM. Borelian $H \subset$ Souslin $H \subset$ Measurable $H$.

4.4. Theorem. Souslin Souslin $H=$ Borelian Souslin $H=$ Souslin $H$.

4.5. THEOREM. If $X$ is a topological space, then Borelian Analytic in $X=$ Analytic in $X$.

4.6. THeOREM. If $X$ is a topological space, then Souslin $K(X) \subset$ Analytic in $X$.

4.7. THEOREM. If $X$ is a Hausdorff space, then Analytic in $X \subset$ Souslin $\mathfrak{F}(X)$.

4.8. THEOREM. If $X$ is a Hausdorff space and $X \in K_{\sigma}(X)$, then Souslin $K(X)=$ Analytic in $X=$ Souslin $\mathfrak{F}(X)$.

4.9. Theorem. If $X$ is a metric space, then Borelian $\mathfrak{F}(X)=$ Borel ring $\mathfrak{F}(X)=$ Borel field $\mathfrak{F}(X)$.

4.10. THEOREM. If $X$ is a complete, separable metric space, then Analytic in $X=$ Souslin $\mathfrak{F}(X)$.

Then following theorem of Sion [8] introduces a property from which are derived necessary and sufficient conditions for open sets to be analytic.

4.11. THEOREM. If $X$ is a Hausdorff space, then $A \sim B \in K_{\sigma}(X)$ whenever $A$ and $B$ belong to $K(X)$ if and only if $A \cap B \in K_{\sigma}(X)$ whenever $A \in K(X)$ and $B \in \mathbb{S}(X)$ if and only if $A \sim B \in$ Analytic in $X$ whenever $A$ and $B$ belong to $K(X)$ if and only if

Borelian $K(X)=$ Borel ring $K(X)$.

The next theorem is an immediate consequence of theorem 4.11.

4.12. THEOREM. If $X$ is a Hausdorff space and $X \in K_{\sigma}(X)$, then $A \sim B \in K_{\sigma}(X)$ whenever $A$ and $B$ belong to $K(X)$ if and only if

$$
\text { (S) }(X) \subset K_{\sigma}(X)
$$


if and only if

Borelian $K(X)=$ Borel ring $K(X)=$ Borel field $K(X)$.

The conclusions of Theorems 4.11 and 4.12 and their recurrence in $\S \S 5$ and 8 motivate the following definition. The terminology is chosen because the condition of interest is a further restriction in kind of a sigma-compact space.

4.13. Definition. $X$ is Borelcompact if and only if $X$ is a topological space, $X \in K_{\sigma}(X)$ and $A \sim B \in K_{\sigma}(X)$ whenever $A$ and $B$ belong to $K(X)$.

Many theorems from here on refer to the cartesian product of a finite number of spaces. In metric spaces the best results are associated with complete, separable spaces. Let us agree that a product of complete, separable, metric spaces is so metrized as to be complete and separable. In topological spaces the best results are associated with Hausdorff spaces which are countable unions of closed compacta. Let us agree that whenever a product of topological spaces is formed the product space is topologized by the standard product topology. That is, a base for the product topology for $X \times Y$ is the family of all cartesian products of open subsets of $X$ and $Y$. At hand then are the next two well-known results.

4.14. THEOREM. If $X$ and $Y$ are Hausdorff spaces, then $X \times Y$ is a Hausdorff space.

4.15. Theorem. If $X \in K_{\sigma}(X)$ and $Y \in K_{\sigma}(Y)$, then

$$
X \times Y \in K_{\sigma}(X \times Y) .
$$

It is essential for our purpose that the topological product of a finite number of Borelcompact spaces be Borelcompact. A sufficient condition for this is given by the following modification of Theorem 3.5 in $[8]$.

4.16. THEOREM. If $X$ and $Y$ are Borelcompact, Hausdorff spaces and there is a countable base for the topology of $X$, then $X \times Y$ is Borelcompact.

For ready reference we combine some parts of 4.12 through 4.16 with 4.3 and 4.8 . 
4.17. THEOREM. If $X$ and $Y$ are Borelcompact, Hausdorff spaces and there is a countable base for the topology of $X$, then $X \times Y$ is Borelcompact and Hausdorff, $\mathbb{B}(X \times Y) \subset$ Souslin $\mathfrak{F}(X \times Y)$ and Borel field $K(X) \subset$ Souslin $\mathfrak{F}(X \times Y)$.

5. Images of Souslin, analytic and measurable sets. The aim of this section is to describe for certain relations a component which maps measurable sets into measurable sets. This is accomplished, in a topological setting, by Definition 5.8.1 and Theorem 5.13. Even though a metric space is not necessarily Borelcompact, the argument leading to Theorem 5.13 can be carried out in a setting of complete, separable, metric spaces. Theorem 5.14 states the metric case.

The component we describe has as one feature the property of being the inverse of a function. Theorem 5.6 gives a condition for counter image with respect to a function to preserve measurability.

The first five theorems listed below are concerned with continuous images and counter images of Souslin and analytic sets. They are well known in their metric space forms. For remarks on the present forms and other closely related results see [2, Section 5].

5.1. Theorem. If $X$ and $Y$ are topological spaces, $A \in$ Analytic in $X$ and $f$ is a continuous function on $A$ to $Y$, then ${ }_{*} f A \in$ Analytic in $Y$.

5.2. THEOREM. If $X$ and $Y$ are Hausdorff spaces, $A \in$ Souslin $K(X)$ and $f$ is a continuous function on $X$ to $Y$, then $* f A \in$ Souslin $K(Y)$.

5.3. THEOREM. If $X$ and $Y$ are Hausdorff spaces, $X \in K_{\sigma}(X)$, $A \in$ Souslin $\mathfrak{F}(X)$ and $f$ is a continuous function on $A$ to $Y$, then

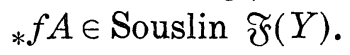

5.4. Theorem. If $X$ and $Y$ are topological spaces, $A \in$ Souslin $\mathfrak{F}(Y)$ and $f$ is a continuous function on $X$ to $Y$, then * $f A \in$ Souslin $\mathfrak{F}(X)$.

5.5. THeoRem. If $X$ and $Y$ are Hausdorff spaces, $X \in K_{\sigma}(X)$, $A \in$ Analytic in $Y$ and $f$ is a continuous function on $X$ to $Y$, then ${ }^{*} f A \in$ Analytic in $X$.

5.6. THEOREM. If $\varphi$ measures $X, \sigma H \in H, f$ is a function with $\operatorname{dmn} f \subset X$ and ${ }^{*} f \alpha \in \operatorname{mbl} \varphi$ whenever $\alpha \in H$, then ${ }^{*} f \alpha \in \operatorname{mbl} \varphi$ when- 
ever $\alpha \in$ Measurable $H$.

Proof. Let $\alpha \in$ Measurable $H$. In view of Definitions 3.4 it is enough to show that

$$
\varphi(T) \geqq \varphi(T \cap * f \alpha)+\varphi(T \sim * f \alpha)
$$

for each $T \subset \operatorname{dmn} \varphi$ with $\varphi(T)<\infty$.

Accordingly let

$$
T \subset \operatorname{dmn} \varphi, \varphi(T)<\infty
$$

and let $\psi$ be the function on the subsets of $\sigma H$ such that for each $\beta \subset \sigma H$

$$
\psi(\beta)=\varphi(T \cap * f \beta) .
$$

It is easy to verify that

$$
\psi \in \operatorname{Mass} H \text {. }
$$

Thus

$$
\alpha \in \operatorname{mbl} \psi
$$

Now by hypothesis

$$
\sigma H \in H
$$

so that

$$
* f \sigma H \in \operatorname{mbl} \varphi \text {. }
$$

Consequently

$$
\begin{aligned}
\varphi(T) & =\varphi\left(T \cap{ }^{*} f \sigma H\right)+\varphi\left(T \sim *^{*} f \sigma H\right) \\
& =\psi(\sigma H)+\varphi\left(T \sim *^{*} \sigma H\right) \\
& =\psi(\alpha)+\psi(\sigma H \sim \alpha)+\varphi\left(T \sim *^{*} f \sigma\right) \\
& =\varphi\left(T \cap{ }^{*} f \alpha\right)+\varphi\left(T \cap{ }^{*} f(\sigma H \sim \alpha)\right)+\varphi\left(T \sim{ }^{*} f \sigma H\right) \\
& =\varphi\left(T \cap{ }^{*} f \alpha\right)+\varphi\left(T \cap{ }^{*} f \sigma H \sim *^{*} \alpha\right)+\varphi\left(T \sim{ }^{*} f \sigma H\right) \\
& \geqq \varphi\left(T \cap{ }^{*} f \alpha\right)+\varphi\left(\left(T \cap{ }^{*} f \sigma H \sim{ }^{*} f \alpha\right) \cup\left(T \sim *^{*} f \sigma H\right)\right) \\
& =\varphi\left(T \cap{ }^{*} f \alpha\right)+\varphi\left(T \sim{ }^{*} f \alpha\right) .
\end{aligned}
$$

Thus ${ }^{*} f \alpha \in \operatorname{mbl} \varphi$ whenever $\alpha \in$ Measurable $H$.

5.7. Corollary. If $\sigma H \in H, f$ is a function with $\operatorname{dmn} f \subset \sigma G$ and ${ }^{*} f \alpha \in$ Measurable $G$ whenever $\alpha \in H$, then ${ }^{*} f \alpha \in$ Measurable $G$ whenever $\alpha \in$ Measurable $H$. 


\subsection{Definitions.}

5.8.1. Singular sections. $\subseteq R$ is the set of pairs in the relation $R$ such that no two different pairs have the same second coordinate.

Thus $\mathfrak{S} R=E(x, y)[R$ is a relation, $(x, y) \in R$ and $* R\{y\}=\{x\}]$.

5.8.2. Plural sections. $\mathfrak{B} R=R \sim \subseteq \mathfrak{S}$, whenever $R$ is a relation.

5.9. THEOREM. If $X$ and $Y$ are Borelcompact, Hausdorff spaces and there is a countable base for the topology of $X$ and $R \in$ Souslin $\mathfrak{\Im}(X \times Y)$, then $\mathfrak{P} R \in$ Souslin $\mathfrak{\Im}(X \times Y)$.

Proof. The hypotheses and Theorem 4.17 ensure that $X \times Y$ and $X \times X \times Y$ are Hausdorff spaces, $X \times Y \in K_{\sigma}(X \times Y)$ and $X \times X \times$ $Y \in K_{\sigma}(X \times X \times Y)$.

Let

$$
C_{1}=E q\left[q=\left(x, x^{\prime}, y\right) \text { for some }\left(x^{\prime}, y\right) \in R \text { and some } x \in X\right]
$$

and

$$
C_{2}=E q\left[q=\left(x, x^{\prime}, y\right) \text { for some }(x, y) \in R \text { and some } x^{\prime} \in X\right] \text {. }
$$

Then both $C_{1}$ and $C_{2}$ are counter images of $R$ under (different) projection functions, and hence by 5.4 belong to Souslin $\mathfrak{\Im}(X \times X \times Y)$. Therefore if

$$
C=C_{1} \cap C_{2} \text {. }
$$

then

$$
C \in \operatorname{Souslin} \mathfrak{F}(X \times X \times Y)
$$

Let

$$
B=E q\left[q=\left(x, x^{\prime}, y\right) \text { and } x \neq x^{\prime} \text { for some } x, x^{\prime} \in X \text { and } y \in Y\right] .
$$

Then

$$
B \in \mathbb{S}(X \times X \times Y) \text { and } B \in \text { Souslin } \mathfrak{F}(X \times X \times Y)
$$

in accordance with 4.17. Now let

$$
A=B \cap C \text {. }
$$

Then 
Finally the image of $A$ under projection into $X \times Y$ is $\Re R$ and by 5.3 belongs to Souslin $\mathfrak{F}(X \times Y)$.

5.10. CoRollary. If $X$ and $Y$ are Borelcompact, Hausdorff spaces and there is a countable base for the topology of $X$ and $R \in$ Souslin $\mathfrak{F}(X \times Y)$, then $\subseteq R \in$ Measurable $\mathfrak{F}(X \times Y)$.

5.11. THEOREM. If $X$ and $Y$ are complete, separable, metric spaces and $R \in$ Souslin $\mathfrak{F}(X \times Y)$, then $\mathfrak{B} R \in$ Souslin $\mathfrak{F}(X \times Y)$ and $\subseteq R \in$ Measurable $\mathfrak{F}(X \times Y)$.

5.12. THEOREM. If $X$ and $Y$ are Borelcompact, Hausdorff spaces and there is a countable base for the topology of $X, R \in$ Souslin $\mathfrak{F}(X \times Y)$ and $A \in$ Souslin $\mathfrak{F}(X)$, then ${ }_{*} \subseteq R A \in$ Measurable $\mathfrak{F}(Y)$.

Proof. In view of 4.17, $X \times Y$ is a Hausdorff space and $X \times Y \in K_{\sigma}(X \times Y)$. The proof is completed in three parts.

Part I. $\operatorname{rng} R \sim \operatorname{rng} \mathfrak{\Re} R=\operatorname{rng} \subseteq R \in$ Measurable $\mathfrak{F}(Y)$.

Proof. The equation is clear. To see that $\mathrm{rng} \subseteq R \in$ Measurable $\mathfrak{F}(Y)$ let $P_{Y}$ be the projection function on $X \times Y$ to $Y$. Then

$$
\operatorname{rng} R={ }_{*} P_{Y} R \in \operatorname{Souslin} \mathfrak{F}(Y) \text {, }
$$

by hypothesis and 5.3, and

$$
\text { rng } \mathfrak{P} R={ }_{*} P_{Y} \mathfrak{\Re} R \in \text { Souslin } \mathfrak{F}(Y),
$$

by 5.9 and 5.3, and the desired result follows with the aid of 4.3.

Part II. ${ }_{*} \subseteq R(A \cap \mathrm{dmn} \subseteq R)={ }_{*} R(A \cap \mathrm{dmn} R) \cap \mathrm{rng} \subseteq R$.

Proof. $\subseteq R$ is the inverse of a function.

Part III. If $A \in$ Souslin $\mathfrak{F}(X)$, then ${ }_{*} \subseteq R A \in$ Measurable $\mathfrak{F}(Y)$.

Proof. Let $P_{X}$ and $P_{Y}$ be the projections functions on $X \times Y$ to $X$ and $Y$ respectively. Then

$$
\operatorname{dmn} R={ }_{*} P_{X} R \in \operatorname{Souslin} \mathfrak{F}(X),
$$

and

$$
A \cap \operatorname{dmn} R \in \text { Souslin } \mathfrak{F}(X) \text {. }
$$




$$
D=(A \cap \operatorname{dmn} R) \times Y .
$$

Then, by 5.4 ,

$$
D=* P_{X}(A \cap \operatorname{dmn} R) \in \operatorname{Souslin} \mathfrak{\Im}(X \times Y) .
$$

Thus

$$
D \cap R \in \operatorname{Souslin} \mathfrak{F}(X \times Y)
$$

Now

$$
{ }_{*} R(A \cap \mathrm{dmn} R)={ }_{*} P_{Y}(D \cap R),
$$

and so by 5.3

$$
{ }_{*} R(A \cap \mathrm{dmn} R) \in \operatorname{Souslin} \mathfrak{F}(Y) .
$$

Accordingly, Parts I and II and Theorem 4.3 yield

$$
\text { *} \Im R(A \cap \mathrm{dmn} R) \in \text { Measurable } \mathfrak{F}(Y) \text {. }
$$

Since

$$
{ }_{*} \subseteq R(A \cap \mathrm{dmn} R)={ }_{*} \subseteq R A
$$

the proof is complete.

5.13. THEOREM. If $X$ and $Y$ are Borelcompact, Hausdorff spaces and there is a countable base for the topology of $X, R \in$ Souslin $\mathfrak{F}(X \times Y)$ and $A \in$ Measurable $\mathfrak{F}(X)$, then ${ }_{*} \subseteq R A \in$ Measurable $\mathfrak{F}(Y)$.

Proof. Let $f=$ inverse $\subseteq R=E(y, x)[(x, y) \in \subseteq R]$. Observe that $f$ is a function, $\operatorname{dmn} f \subset Y=\sigma \mathfrak{F}(Y)$, $\operatorname{rng} f \subset X=\sigma \mathfrak{F}(X)$ and ${ }^{*} f A=$ * $\subseteq R A$. Now application of Theorems 5.12 and 5.7 yields the desired conclusion.

5.14. THEOREM. If $X$ and $Y$ are complete, separable, metric spaces, $R \in$ Souslin $\mathfrak{F}(X \times Y)$ and $A \in$ Measurable $\mathfrak{F}(X)$, then ${ }_{*} \subseteq R A \in$ Measurable $\mathfrak{F}(Y)$.

6. Extracted, derived and removed sets. In this section we introduce three transfinite set operations which are employed later to extend the results of $\S 5$.

\subsection{DEFINITIONS.}

6.1.1. Extracted set. $\mathscr{G} R \mathscr{B}=\cup_{G \in \mathscr{B}} \mathfrak{S}(R \cap G)$.

6.1.2. Extracted set of order $\alpha$. 
For $R$ a relation, $\mathscr{B}$ a family of sets and $\alpha \in Q$ let extr $R \mathscr{B} \alpha$ be $f(\alpha)$ where $f$ is the function on $Q$ for which

$$
f(\beta)=\bigcup_{\xi \in \beta} f(\xi) \cup \mathfrak{F}\left(R \sim \bigcup_{\xi \in \beta} f(\xi)\right) \mathscr{B}
$$

whenever $\beta \in Q$.

Thus one has the inductive formulas:

$$
\operatorname{extr} R \mathscr{B} 0=\mathbb{E} R \mathscr{B}
$$

and

$$
\operatorname{extr} R \mathscr{B} \alpha=\bigcup_{\xi \in \infty} \operatorname{extr} R \mathscr{B} \xi \cup \mathscr{F}\left(R \sim \bigcup_{\xi \in \infty} \operatorname{extr} R \mathscr{B} \xi\right) \mathscr{B}
$$

whenever $\alpha \in Q$.

The following two theorems are easily proved.

6.2. THEOREM. If $\alpha \in Q$, then

$$
\operatorname{extr} R \mathscr{B} \alpha=\bigcup_{\xi \in \bar{\alpha}} \mathfrak{F}\left(R \sim \bigcup_{\eta \in \xi} \operatorname{extr} R \mathscr{B} \eta\right) \mathscr{B}
$$

6.3. THEOREM. If $\alpha \in Q$, then extr $R \mathscr{B} \alpha \subset R$.

6.4. Definitions.

6.4.1. Derived set.

DD $X A$ is the set of accumulation points of $A$, whenever $X$ is a topological space and $A \subset X$.

6.4.2. Derived set of order $\alpha$.

For $X$ a topological space, $A \subset X$ and $\alpha \in Q$ let $\operatorname{drv} X A \alpha$ be $f(\alpha)$ where $f$ is the function on $Q$ for which

$$
f(\beta)=A \cap \mathfrak{D} X\left(A \cap \bigcap_{\xi \in \beta} f(\xi)\right)
$$

whenever $\beta \in Q$.

Thus one has the inductive formulas:

$$
\operatorname{drv} X A 0=A \cap \mathfrak{D} X A
$$

and

$$
\operatorname{drv} X A \alpha=A \cap \mathfrak{D} X\left(A \cap \bigcap_{\xi \in \alpha} \mathrm{drv} X A \xi\right)
$$

whenever $\alpha \in Q$.

6.5. Definitions.

6.5.1. Isolated set. $\mathfrak{X} X=A \sim \mathfrak{D} X A$ 
6.5.2. Removed set of order $\alpha$.

For $X$ a topological space, $A \subset X$ and $\alpha \in Q$ let $\operatorname{rmv} X A \alpha$ be $f(\alpha)$ where $f$ is the function on $Q$ for which

$$
f(\beta)=\bigcup_{\xi \in \beta} f(\xi) \cup \Im X\left(A \sim \bigcup_{\xi \in \beta} f(\xi)\right)
$$

whenever $\beta \in Q$.

Thus one has the inductive formulas:

$$
\operatorname{rmv} X A 0=\mathfrak{Y} X A
$$

and

$$
\operatorname{rmv} X A \alpha=\bigcup_{\xi \in \infty} \operatorname{rmv} X A \xi \cup \Im X\left(A \sim \bigcup_{\xi \in \infty} \operatorname{rmv} X A \xi\right)
$$

whenever $\alpha \in Q$.

The following two lemmas are easily proved.

6.6. Lemma. If $X$ is a topological space, $A \subset X$ and $\alpha \in \beta \in Q$, then $A \supset \operatorname{drv} X A \alpha \supset \operatorname{drv} X A \beta$.

6.7. Lemma. If $X$ is a topological space, $A \subset X$ and $\alpha \in Q$, then

$$
\operatorname{rmv} X A \alpha=\bigcup_{\eta \in \bar{\alpha}} \mathfrak{\Im} X\left(A \sim \bigcup_{\xi \in \eta} \operatorname{rmv} X A \xi\right)
$$

and

$$
\operatorname{rmv} X A \alpha \subset A
$$

6.8. THEOREM. If $X$ is a topological space, $A \subset X$ and $\alpha \in Q$, then

$$
\operatorname{rmv} X A \alpha=A \sim \operatorname{drv} X A \alpha
$$

and

$$
\operatorname{drv} X A \alpha=A \sim \operatorname{rmv} X A \alpha
$$

Proof. In view of 6.6 and 6.7 the conjuncts of the conclusion are equivalent. Theorefore it is enough to prove

$$
\operatorname{rmv} X A \alpha=A \sim \operatorname{drv} X A \alpha .
$$

The proof is by induction.

Clearly

$$
\operatorname{rmv} X A 0=A \sim \mathrm{drv} X A 0 .
$$


Let $\beta \in Q$ and suppose that for each $\eta \in \beta$

$$
\operatorname{rmv} X A \eta=A \sim \operatorname{drv} X A \eta \text {. }
$$

Then also for each $\eta \in \beta$

$$
\operatorname{drv} X A \eta=A \sim \operatorname{rmv} X A \eta .
$$

The proof will be complete when the following argument is verified.

$$
\begin{aligned}
\operatorname{rmv} X A \beta & =\bigcup_{\eta \in \bar{\beta}} \Im X\left(A \sim \bigcup_{\xi \in \eta} \operatorname{rmv} X A \xi\right) \\
& =\bigcup_{\eta \in \bar{\beta}} \Im X\left(A \cap\left(A \sim \bigcup_{\xi \in \eta} \operatorname{rmv} X A \xi\right)\right) \\
& =\bigcup_{\in \in \bar{\beta}} \Im X\left(A \cap \bigcap_{\xi \in \eta}(A \sim \operatorname{rmv} X A \xi)\right) \\
& =\bigcup_{\eta \in \bar{\beta}} \Im X\left(A \cap \bigcap_{\xi \in \eta} \operatorname{drv} X A \xi\right) \\
& =\bigcup_{\ell \in \bar{\beta}}\left(A \cap \bigcap_{\xi \in \eta} \operatorname{drv} X A \xi \sim \mathfrak{D} X\left(A \cap \bigcap_{\xi \in \eta} \operatorname{drv} X A \xi\right)\right) \\
& =\bigcup_{\eta \in \bar{\beta}}\left(A \cap \bigcap_{\xi \in \eta} \operatorname{drv} X A \xi \sim\left(A \cap \mathfrak{D} X\left(A \cap \bigcap_{\xi \in \eta} \operatorname{drv} X A \xi\right)\right)\right) \\
& =\bigcup_{\eta \in \bar{\beta}}\left(A \cap \bigcap_{\xi \in \eta} \operatorname{drv} X A \xi \sim \operatorname{drv} X A \eta\right) \\
& =\bigcup_{\eta \in \bar{\beta}}\left(A \cap \bigcap_{\xi \in \eta} \operatorname{drv} X A \xi \sim(A \cap \operatorname{drv} X A \eta)\right) \\
& =A \sim(A \cap \operatorname{drv} X A \beta) \\
& =A \sim \operatorname{drv} X A \beta .
\end{aligned}
$$

There are ten steps in the argument above which are now verified in order: (i) Lemma 6.7. (ii) Lemma 6.7. (iii) De Morgan's Law. (iv) The induction hypothesis. (v) Definition 6.5.1. (vi) Set theory. (vii) Definition 6.4.2. (viii) Lemma 6.6. (ix) Because of lemma 6.6 the union telescopes. (x) Lemma 6.6.

7. Subvalent Relations. The purpose of this section is realized in Theorem 7.6 which states that a subvalent relation (Definition 7.2) is an extracted set of countable order with respect to a (not necessarily countable) base for the topology of the space containing the relation. When in the concluding section we recall the hypotheses of Theorem 5.13 and assume each topology involved has a countable base, we obtain the result (Theorem 8.3) that a relation which is both a Souslin set and subvalent preserves measurability. The corresponding extension of Theorem 5.14 is stated in Theorem 8.4.

7.1. Definition. Horizontal sections.

$\mathfrak{g} R y$ is the set of pairs in the relation $R$ which have second coordinate $y$. Thus 


$$
\mathfrak{g} R y=E(x, t)[R \text { is a relation, }(x, t) \in R \text { and } t=y] \text {. }
$$

7.2. Definition. Subvalent relations.

Subvalent $Z$ is the family of all relations $R$ such that $Z$ is a topological product space, $R \subset Z$ and for some countable ordinal $\alpha$

$$
\operatorname{drv} Z \mathfrak{S} R y \alpha=0
$$

for each $y$ in the second coordinate space of $Z$.

7.3. Lemma. If $R$ is a relation, $\operatorname{rng} R \subset B$ and $T \subset R$ for each $T \in \mathscr{T}$, then

$$
\mathfrak{S} R y \sim \bigcup_{T \in \mathscr{S}} \mathfrak{S} T y=\mathfrak{E}\left(R \sim \bigcup_{T \in \mathscr{T}} \bigcup_{t \in B} \mathfrak{S} T t\right) y .
$$

Proof. Clearly, if $R$ is a relation and $T \subset R$ for each $T \in \mathscr{T}$, then $\mathbf{U}_{T \in \mathscr{F}} \mathfrak{T} T y=\mathfrak{g}_{\sigma} \mathscr{T} y$. Again, if $R$ is a relation and $T \subset R$, then $\mathfrak{S} R y \sim \mathfrak{S} T y=\mathfrak{g}(R \sim T) y$. Moreover, under the hypotheses, $\sigma \mathscr{T} \subset R$ and, for each $T \in \mathscr{T}, T=\bigcup_{t \in B} \mathfrak{T} T t$. Consequently,

$$
\begin{aligned}
\mathfrak{S} R y \sim \bigcup_{T \in \mathscr{S}} \mathfrak{S} T y & =\mathfrak{S} R y \sim \mathfrak{S}_{\sigma} \sigma y \\
& =\mathfrak{Q}(R \sim \sigma \mathscr{T}) y \\
& =\mathfrak{S}\left(R \sim \bigcup_{T \in \mathscr{T}} T\right) y \\
& =\mathfrak{E}\left(R \sim \bigcup_{T \in \mathscr{S}} \bigcup_{t \in B} \mathfrak{S} T t\right) y .
\end{aligned}
$$

7.4. Lemma. If $Z$ is a topological product space, $R \subset Z, \alpha \in Q$ and $Y$ is the second coordinate space of $Z$, then

$$
\mathfrak{S} R y \sim \bigcup_{\eta \in \infty} \mathrm{rmv} Z \mathfrak{S}_{\mathfrak{C}} R y \eta=\mathfrak{S}\left(R \sim \bigcup_{\eta \in \infty} \bigcup_{t \in Y} \operatorname{rmv} Z \mathfrak{S}_{\mathfrak{S}} R t \eta\right) y .
$$

Proof. Let

$$
\mathscr{T}=E T[T=\operatorname{rmv} Z H R y \eta \text { for some } \eta \in \alpha] \text {. }
$$

By 6.7 and 7.1

$$
T \subset R \text { for each } T \in \mathscr{T} \text {. }
$$

Thus 7.4 follows from 7.3.

7.5. THEOREM. If $Z$ is a topological product space, $R \subset Z, \mathscr{B}$ is a base for the topology of $Z, \alpha \in Q$ and $Y$ is the second coordinate space of $Z$, then

$$
\bigcup_{y \in Y} \operatorname{rmv} Z \mathfrak{C} R y \alpha=\operatorname{extr} R \mathscr{B} \alpha
$$


and

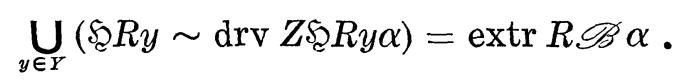

Proof. In view of 6.8 the conjuncts of the conclusion are equivalent. Therefore it is enough to prove

$$
\bigcup_{y \in Y} \operatorname{rmv} Z \mathfrak{g}_{\mathfrak{2}} R y \alpha=\operatorname{extr} R \mathscr{B} \alpha .
$$

The proof is by induction.

It is immediate from the definition of a base for a topology that

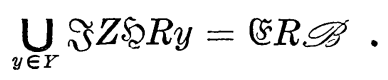

Thus

$$
\bigcup_{y \in Y} \operatorname{rmv} Z S_{\mathscr{C}} R y 0=\operatorname{extr} R \mathscr{B} 0 \text {. }
$$

Let $\beta \in Q$ and suppose that for each $\eta \in \beta$

$$
\bigcup_{y \in Y} \operatorname{rmv} Z \$_{\mathfrak{C}} R y \eta=\operatorname{extr} R \mathscr{B} \eta \text {. }
$$

The proof will be complete when the following argument is verified:

$$
\begin{aligned}
& \bigcup_{y \in Y} \operatorname{rmv} Z \mathfrak{S}_{\mathfrak{C}} R y \beta \\
& =\bigcup_{y \in Y}\left(\bigcup_{\eta \in \beta} \operatorname{rmv} Z \mathfrak{S}_{2} R y \eta \cup \Im Z\left(\mathfrak{S} R y \sim \bigcup_{\eta \in \beta} \operatorname{rmv} Z \mathfrak{S}_{2} R y \eta\right)\right)
\end{aligned}
$$

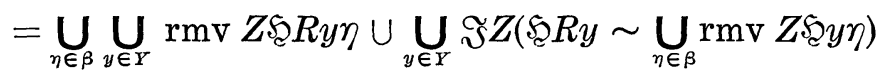

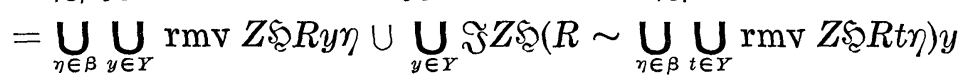

$$
\begin{aligned}
& =\bigcup_{\eta \in \beta} \operatorname{extr} R \mathscr{B} \eta \cup \bigcup_{y \in Y} \Im Z \mathscr{C}\left(R \sim \bigcup_{\eta \in \beta} \operatorname{extr} R \mathscr{B} \eta\right) y \\
& =\bigcup_{\eta \in \beta} \operatorname{extr} R \mathscr{B} \eta \cup \mathscr{F}\left(R \sim \bigcup_{\eta \in \beta} \operatorname{extr} R \mathscr{B} \eta\right) \mathscr{B} \\
& =\operatorname{extr} R \mathscr{B} \beta \text {. }
\end{aligned}
$$

There are six steps in the argument above which are now verified in order: (i) Definition 6.5.2. (ii) Set theory. (iii) Lemma 7.4. (iv) The induction hypothesis. (v) (7.5.1). (vi) Definition 6.1.2.

7.6. THEOREM. If $Z$ is a topological product space, $R \in$ Subvalent $Z$ and $\mathscr{B}$ is a base for the topology of $Z$, then for some countable ordinal $\alpha$

$$
R=\operatorname{extr} R \mathscr{B} \alpha
$$


Proof. Apply 7.2 to 7.5.

8. Images of Measurable sets.

8.1. THEOREM. If $X$ and $Y$ are Borelcompact, Hausdorff spaces and there is a countable base for the topology of $X, R \in$ Souslin $\mathfrak{F}(X \times Y), \mathscr{B} \subset \mathbb{S}(X \times Y)$ and $\mathscr{B}$ is countable, $\alpha \in \Omega$ and $A \in$ Measurable $\mathfrak{F}(X)$, then ${ }_{*}$ extr $R \mathscr{B} \alpha A \in$ Measurable $\mathfrak{F}(Y)$.

The proof is in six parts.

Part I. $\quad R \sim G \in$ Souslin $\mathfrak{F}(X \times Y)$ and $\mathfrak{P}(R \cap G) \in$ Souslin $\mathfrak{F}(X \times Y)$ whenever $G \in \mathscr{B}$.

Proof. Apply Theorems 4.17 and 5.9.

Part II. $R \sim \mathscr{S} R \mathscr{B} \in$ Souslin $\mathfrak{F}(X \times Y)$.

Proof. Recall Definitions 5.8 and 6.1.1 to see that

$$
\begin{aligned}
R \sim \mathfrak{S} R \mathscr{P} & =R \sim \bigcup_{G \in \mathscr{B}} \subseteq(R \cap G) \\
& =R \cap \bigcap_{G \in \mathscr{B}}((R \sim G) \cup \mathfrak{P}(R \cap G)) .
\end{aligned}
$$

Thus

$$
R \sim \mathfrak{F} R \mathscr{B} \in \text { Souslin } \mathfrak{F}(X \times Y)
$$

in accordance with Part I, since $\mathscr{B}$ is countable.

Part III. $R \sim \operatorname{extr} R \mathscr{B} \alpha \in$ Souslin $\mathfrak{F}(X \times Y)$.

Proof. The statement holds by mathematical induction in view of Part II, the fact that $\alpha$ is countable and the following formula:

$$
\begin{aligned}
R & \sim \operatorname{extr} R \mathscr{B} \alpha \\
& =R \sim\left(\bigcup_{\eta \in \infty} \operatorname{extr} R \mathscr{B} \eta \cup \mathscr{F}\left(R \sim \bigcup_{\eta \in \infty} \operatorname{extr} R \mathscr{B} \eta\right) \mathscr{B}\right) \\
& =R \sim \bigcup_{\eta \in \infty} \operatorname{extr} R \mathscr{B} \eta \sim \mathscr{F}\left(R \sim \bigcup_{\eta \in \infty} \operatorname{extr} R \mathscr{B} \eta\right) \mathscr{B} \\
& =R \cap \bigcap_{\eta \in \infty}(R \sim \operatorname{extr} R \mathscr{B} \eta) \sim \mathfrak{F}\left(R \cap \bigcap_{\eta \in \infty}(R \sim \operatorname{extr} R \mathscr{B} \eta)\right) \mathscr{B} .
\end{aligned}
$$

Part IV. $\quad R \sim \bigcup_{\eta \in \infty} \operatorname{extr} R \mathscr{B} \eta \in$ Souslin $\mathfrak{F}(X \times Y)$

Proof. $\quad R \sim \bigcup_{\eta \in \infty} \operatorname{extr} R \mathscr{B} \eta=R \cap \bigcap_{\eta \in \alpha}(R \sim \operatorname{extr} R \mathscr{B} \eta)$, and the result follows from Part III because $\alpha$ is countable. 
Part V. * $[\tilde{S} \mathscr{B} A \in$ Measurable $\mathfrak{F}(Y)$.

Proof. For any set $A,{ }_{*}\left[\mathscr{B} \mathscr{B} A=\bigcup_{G \in \mathscr{B}} \mathscr{S}(R \cap G) A\right.$. Hence the result follows from 5.13 because $\mathscr{B}$ is countable.

Part VI. ${ }_{*} \operatorname{extr} R \mathscr{B} \alpha A \in$ Measurable $F(Y)$.

Proof. From 6.2

$$
\operatorname{extr} R \mathscr{B} \alpha=\bigcup_{\xi \in \mathscr{a}} \mathfrak{F}\left(R \sim \bigcup_{\eta \in \xi} \operatorname{extr} R \mathscr{B} \eta\right) \mathscr{B},
$$

and by part IV

$$
R \sim \bigcup_{\eta \in \xi} \operatorname{extr} R \mathscr{B} \eta \in \text { Souslin } \mathfrak{F}(X \times Y) .
$$

The desired result now follows from Part V.

8.2. THEOREM. If $X$ and $Y$ are complete, separable, metric spaces, $R \in$ Souslin $\mathfrak{F}(X \times Y), \mathscr{B} \subset \mathbb{B}(X \times Y)$ and $\mathscr{B}$ is countable, $\alpha \in \Omega$ and $A \in$ Measurable $\mathfrak{F}(X)$, then ${ }_{*}$ extr $R \mathscr{B} \alpha A \in$ Measurable $\mathfrak{F}(Y)$.

8.3. THEOREM. If $X$ and $Y$ are Borelcompact, Hausdorff spaces, there is a countable base for the topology of $X \times Y$ and $R \in$ Souslin $\mathfrak{F}(X \times Y) \cap$ Subvalent $(X \times Y)$, then ${ }_{*} R A \in$ Measurable $\mathfrak{F}(Y)$ whenever $A \in$ Measurable $\mathfrak{\Im}(X)$.

Proof. There are countable bases for the topologies of $X$ and $Y$ separately, hence the theorem follows from 7.6 and 8.1.

8.4. THEOREM. If $X$ and $Y$ are complete separable metric spaces and $R \in$ Souslin $\mathfrak{F}(X \times Y) \cap$ Subvalent $(X \times Y)$, then ${ }_{*} R A \in$ Measurable $\mathfrak{F}(Y)$ whenever $A \in$ Measurable $\mathfrak{F}(X)$.

Proof. Theorems 7.6 and 8.2 .

8.5. REMARKS. Let

Zero Measurable $H$ be the family of all sets $A$ such that

$$
\varphi(A)=0
$$

for each $\varphi$ which measures $\sigma H$ and for which $H \subset \operatorname{mbl} \varphi, \varphi(\sigma H)<\infty$ and $\varphi(B)=0$ whenever $B$ is a countable subset of $\sigma H$.

A.S. Besicovitch [1] has shown that for $X$ a complete, separable, metric space there exist noncountable sets in Zero Measurable $\mathfrak{F}(X)$. 
Consequently the following extension of Theorem 8.4 is of some interest.

THeOREM. If $X$ and $Y$ are complete, separable, metric spaces, $R \in$ Souslin $\mathfrak{F}(X \times Y)$ and $\left(R \sim \bigcup_{u \in H} \mathfrak{g} R y\right) \in$ Subvalent $(X \times Y)$ for some $H \in$ Zero Measurable $\mathfrak{F}(Y)$, then ${ }_{*} R A \in$ Measurable $\mathfrak{F}(Y)$ whenever $A \in$ Measurable $\mathfrak{F}(X)$.

8.6. REMARKS. M. Sion [6] has proved several theorems concerning images of measurable sets. The following theorem is representative of his results:

THEOREM. If $X$ and $Y$ are complete, separable, metric spaces, $f$ is a continuous function on $X$ to $Y$ and $E y\left[y \in \operatorname{rng} f\right.$ and ${ }^{*} f\{y\}$ is not countable $] \in$ Zero Measurable $\mathfrak{F}(Y)$, then ${ }_{*} f A \in$ Measurable $\mathfrak{F}(Y)$ whenever $A \in$ Measurable $\mathfrak{F}(X)$.

\section{REFERENCES}

1. A.S. Besicovitch, Concentrated and rarified sets of points, Acta Math. 62 (1934), 289-300.

2. D.W. Bressler and M. Sion, The current theory of analytic sets, Canad. J. Math., 16 (1964), 207-230.

3. G. Choquet, Theory of capacities, Ann. Inst. Fourier, Grenoble, 5 (1953-1954), 131-295.

4. N. Lusin, Sur la classification de M. Baire, C.R. Acad. Sci. Paris, 164 (1917), 91-94.

5. N. Lusin et W. Sierpiński, Sur quelques propriétés des ensembles ( $\mathscr{A})$, Bull. Acad. Sci. Cracovie (1918), 37-48.

6. M. Sion, Variational measure, Trans. Amer. Math. Soc. 83 (1956), 205-221.

7. - On analytic sets in topological spaces, Trans. Amer. Math. Soc., 96 (1960), 341-354.

8. - Continuous images of Borel sets, Proc. Amer. Math. Soc. 12 (1961), 385391.

9. M. Souslin, Sur une définition des ensembles mesurables $B$ sans nombres transfinis, C.R. Acad. Sci. Paris, 164 (1917), 88-91.

Received July 25, 1964. 



\section{PACIFIC JOURNAL OF MATHEMATICS}

H. SAMELSON

Stanford University Stanford, California

R. M. BLUMENTHAL

University of Washington

Seattle, Washington 98105

\section{EDITORS}

\author{
*J. DugundJI \\ University of Southern California \\ Los Angeles, California 90007 \\ RICHARD ARENS \\ University of California \\ Los Angeles, California 90024
}

\section{ASSOCIATE EDITORS}

E. F. BeCKENBACH

B. H. NEUMANN

F. WOLF

K. YosidA

\section{SUPPORTING INSTITUTIONS}

UNIVERSITY OF BRITISH COLUMBIA

CALIFORNIA INSTITUTE OF TECHNOLOGY

UNIVERSITY OF CALIFORNIA

MONTANA STATE UNIVERSITY

UNIVERSITY OF NEVADA

NEW MEXICO STATE UNIVERSITY

OREGON STATE UNIVERSITY

UNIVERSITY OF OREGON

OSAKA UNIVERSITY

UNIVERSITY OF SOUTHERN CALIFORNIA
STANFORD UNIVERSITY

UNIVERSITY OF TOKYO

UNIVERSITY OF UTAH

WASHINGTON STATE UNIVERSITY

UNIVERSITY OF WASHINGTON

AMERICAN MATHEMATICAL SOCIETY CHEVRON RESEARCH CORPORATION TRW SYSTEMS

NAVAL ORDNANCE TEST STATION 


\section{Pacific Journal of Mathematics}

Vol. 18, No. 1

March, 1966

Edward Joseph Barbeau, Semi-algebras that are lower semi-lattices ...... 1

Steven Fredrick Bauman, The Klein group as an automorphism group

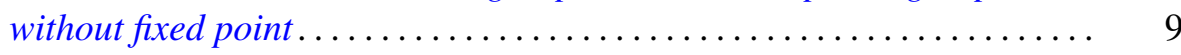

Homer Franklin Bechtell, Jr., Frattini subgroups and $\Phi$-central groups .... 15

Edward Kenneth Blum, A convergent gradient procedure in prehilbert

spaces ............................................

Edward Martin Bolger, The sum of two independent exponential-type random variables ...................................

David Wilson Bressler and A. P. Morse, Images of measurable sets .......

Dennison Robert Brown and J. G. LaTorre, A characterization of uniquely

divisible commutative semigroups........................

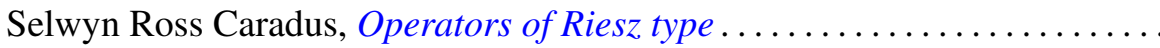

Jeffrey Davis and Isidore Isaac Hirschman, Jr., Toeplitz forms and ultraspherical polynomials ............................

Lorraine L. Foster, On the characteristic roots of the product of certain rational integral matrices of order two ......................

Alfred Gray and S. M. Shah, Asymptotic values of a holomorphic function

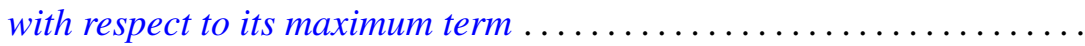

Sidney (Denny) L. Gulick, Commutativity and ideals in the biduals of

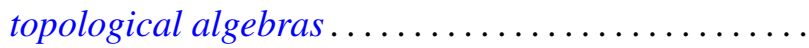

G. J. Kurowski, Further results in the theory of monodiffric functions

Lawrence S. Levy, Commutative rings whose homomorphic images are self-injective .

Calvin T. Long, On real numbers having normality of order $k$....

Bertram Mond, An inequality for operators in a Hilbert space. ...

John William Neuberger, The lack of self-adjointness in three-point boundary value problems ........................

C. A. Persinger, Subsets of $n$-books in $E^{3}$

Oscar S. Rothaus and John Griggs Thompson, A combinatorial problem in the symmetric group ............................... 175

Rodolfo DeSapio, Unknotting spheres via Smale .................. 179

James E. Shockley, On the functional equation

$$
F(m n) F((m, n))=F(m) F(n) f((m, n)) \ldots \ldots \ldots
$$

Kenneth Edward Whipple, Cauchy sequences in Moore spaces ... 